PRL-TH-97/24

hep-ph/9708332

\title{
CP-violating dipole form factors of the top quark and tau lepton in scalar leptoquark models
}

\author{
P. Poulose ${ }^{a}$ and Saurabh D. Rindani ${ }^{a, b}$ \\ ${ }^{a}$ Theory Group, Physical Research Laboratory \\ Navrangpura, Ahmedabad 380 009, India \\ ${ }^{b}$ Instituto de Física Corpuscular - CSIC \\ Departament de Física Teòrica \\ Universitat de València, 46100 Burjassot (València), Spain
}

\begin{abstract}
We calculate the $C P$-violating electric and weak dipole form factors of the top quark and the tau lepton in models with scalar leptoquarks coupling only to the third generation of quarks and leptons. We obtain numerical values of the real and imaginary parts of these form factors at various energies for different values of leptoquark masses and couplings. The existing limits on the tau electric and weak dipole form factors allows us to put a limit on the masses and couplings of such leptoquarks and therefore on the top electric and weak dipole form factors. We also discuss constraints on the form factors coming from indirect limits on leptoquark masses and couplings deduced from LEP results on $Z$ properties.
\end{abstract}

PACS Nos. 11.30.Er, 13.40.Em, 14.60.Fg, 14.65.Ha

Keywords: CP violation, scalar leptoquarks, dipole moments, top quark, tau lepton 


\section{Introduction}

The standard model (SM) of electroweak interactions predicts that $C P$ violation outside the $K$ - and $B$-meson systems would be unobservably small. Thus, if any $C P$ violation is observed in the future outside of these systems, it would be a signal of new physics. In particular, the observation of electric dipole moments of elementary particles would signal new mechanisms of $C P$ violation lying beyond SM, since SM predicts unobservably small electric dipole moments. Likewise, their generalization to $Z$ couplings (the so-called weak dipole moments), as well as to couplings at nonzero momentum transfers, viz., dipole form factors (DFF), would also serve as signals for $C P$ violation beyond SM.

Recent experiments at the Large Electron Positron Collider (LEP) at CERN have established an upper bound on the weak dipole form factor of the tau lepton at the $Z$ peak, by looking for $C P$-violating momentum correlations [1, 2]. The latest result is from the OPAL collaboration [2], who have obtained the limits

$$
\begin{aligned}
& \operatorname{Re} d_{\tau}^{Z}<5.6 \times 10^{-18} e \mathrm{~cm} \\
& \operatorname{Im} d_{\tau}^{Z}<1.5 \times 10^{-17} e \mathrm{~cm} .
\end{aligned}
$$

It is expected that better limits would be obtained in future experiments using longitudinally polarized electrons [3]. For example, it was shown that using certain $C P$-odd vector correlations, it would be possible to measure an EDFF of $\tau$ of the order of $10^{-19} e \mathrm{~cm}$ [3].

Estimates have also been made of the senstivity to which various experiments at $e^{+} e^{-}$[4] as well as hadron colliders [5] might be able to measure top electric and weak dipole form factors.

Most models seem to predict values of electric dipole form factors (EDFF) and weak dipole form factors (WDFF) an order of magnitude below the observable level in the next generation of planned experiments. In this situation, it is a worthwhile pursuit to look at other models which might predict large DFF's which could be tested in the near future.

In this paper we consider the possibility that models with relatively light scalars with complex couplings to a third-generation lepton-quark pair can give rise to EDFF and WDFF of third generation fermions, viz., the top quark and the tau lepton, at a significant level. This possibility for the tau 
lepton has been considered recently by Mahanta [6] and Bernreuther et al. [0]. While we confirm some results for tau of [6] and [7], we also obtain new results for the CP-violating form factors of the top quark, and treat the tau and top cases in a concerted manner. We have also studied the implications of the existing experimental bounds from LEP on the weak dipole form factor of $\tau$ at the $Z$ resonance, and on the masses and couplings of leptoquarks from loop effects contributing to the $Z$ partial widths.

A large number of extensions of SM predict the existence of colour triplet particles carrying simultaneously lepton and baryon number, called leptoquarks. These models include grand unified theories, technicolour models, superstring inspired models and composite models. Without reference to specific models, the masses and couplings of leptoquarks can be constrained using low-energy experiments. These experiments test predictions of leptoquark interactions for atomic parity violation, meson decay, flavour-changing neutral currents and meson-antimeson mixing.

There have been several direct searches for leptoquarks at high energy accelerators. At the Large Electron-Positron collider (LEP) at CERN, a lower bound of $45-73 \mathrm{GeV}$ for the mass of leptoquarks was put [8]. The limit coming from $p \bar{p}$ colliders is $175 \mathrm{GeV}$ from D0 [9] and $131-133 \mathrm{GeV}$ from CDF [10] on the mass of a scalar leptoquark decaying into an electron-jet pair. On the mass of the third generation scalar leptoquark decaying into $b \tau$, CDF has given a bound of $99 \mathrm{GeV}$ [10] and a bound of $80 \mathrm{GeV}$ was obtained by D0 for such a leptoquark decaying into $b \nu_{\tau}$ [9]. The excess of large- $Q^{2}$ events reported in $e^{+} p$ collisions at HERA [11] have been interpreted as due to leptoquark production [12], the mass of the leptoquark being around 200 $\mathrm{GeV}$. The earlier limit coming from HERA is dependendent on the leptoquark type and couplings, and the lower bound is between 92 and $184 \mathrm{GeV}$ [13]. Bounds possible at future $p p, e p, e^{+} e^{-}, e \gamma$ and $\gamma \gamma$ experiments has been a topic of serious study.

Indirect bounds on masses and couplings can be obtained from the results of low-energy experiments [14]. However, these constraints are strong only for leptoquarks that couple to quarks and leptons of the first and second generations.

While there are strong constraints on masses of leptoquarks which also couple to pairs of quarks, thus violating baryon number as well as lepton number, the constraints on the couplings and masses of leptoquarks which do not couple to two quarks are weaker. Moreover, these constraints are 
strongest for the first and second generations, and considerably weaker for leptoquarks coupling only to the third generation of quarks and leptons.

Strong constraints on leptoquarks which couple to leptons and quarks of the third generation have been obtained from their contributions to the radiative corrections to $Z$ properties [15, 16, 17. The authors of 115 have studied vertex corrections to the leptonic partial widths of the $Z$ induced by leptoquark loops and obtained stringent constraints on leptoquark masses and couplings. The authors of [16, 17] performed a global fit to the LEP data including contributions from a scalar leptoquark loop. They also arrive at stringent constraints on leptoquark masses and couplings.

Earlier work on $\mathrm{CP}$ violation in leptoquark models can be found in 18 , 19.

In this paper, we calculate the EDFF and WDFF for the third generation fermions, viz., the top quark and the tau lepton, in scalar leptoquark models within the context of an $S U(2)_{L} \times U(1) \times S U(3)_{c}$ gauge theory, where the leptoquarks couple to only the third generation fermions. We then use the indirect limits obtained from LEP on the masses and couplings of the leptoquark to investigate what are the possible values of the EDFF and WDFF in these models consistent with these limits.

Briefly, our results are as follows. The present experimental limits on $\tau$ EDFF and WDFF do not put stringent constraints on masses and couplings of leptoquarks. Consequently, these results are consistent with a top EDFF of the order of $10^{-19} \mathrm{e} \mathrm{cm}$, and a top WDFF of the order of $10^{-20} \mathrm{e} \mathrm{cm}$. The indirect LEP limits, however, constrain these form factors to be at least three orders of magnitude lower. They also give limits on tau form factors about two orders of magnitude lower than the direct experimental limits.

We describe in the next section couplings of scalar leptoquarks with various transformation properties under $S U(2)_{L} \times U(1) \times S U(3)_{c}$. In Section 3, we give expressions for the imaginary parts of the EDFF's and WDFF's of $\tau$ and $t$ arising from these leptoquark couplings, and write dispersion relations for obtaining the corresponding real parts. In Section 4 we present numerical results and the last section (Section 5) contains our conclusions. 


\section{Scalar leptoquark couplings}

We will describe in this section leptoquark couplings in an $S U(2)_{L} \times U(1) \times$ $S U(3)_{c}$ gauge theory, assuming that baryon-number violating couplings to diquarks are somehow forbidden, as required by strong bounds on protondecay searches. In that case, the only possible leptoquark representations which could have couplings to the standard-model representations of quarks and leptons are as shown in Table 1, together with their quantum numbers [20].

The most general Lagrangian containing all possible forms of couplings of scalar leptoquarks to a lepton and quark pair is given by

$$
\mathcal{L}_{\text {eff }}=\mathcal{L}_{F=2}+\mathcal{L}_{F=0},
$$

where

$$
\mathcal{L}_{F=0}=h_{2 L} \overline{u_{R}} R_{2}^{T} i \tau_{2} l_{L}+h_{2 R} \overline{q_{L}} e_{R} R_{2}+\tilde{h}_{2 L} \overline{d_{R}} \tilde{R}_{2}^{T} i \tau_{2} l_{L}+\text { h.c. },
$$

and

$\mathcal{L}_{F=2}=\left(g_{1 L} \overline{q_{R}^{c}} i \tau_{2} l_{L}+g_{1 R} \overline{u_{L}^{c}} e_{R}\right) S_{1}+\tilde{g}_{1 R} \overline{d_{L}^{c}} e_{R} \tilde{S}_{1}+g_{3 L} \overline{q_{R}^{c}} i \tau_{2} \vec{\tau} l_{L} \cdot \overrightarrow{S_{3}}+$ h.c.,

The two pieces correspond to the fermion number $F=0$ for the leptoquarks $R_{2}$ and $\tilde{R}_{2}$, and $F=-2$ for $S_{1}, \tilde{S}_{1}$ and $S_{3}$. Colour indices are suppressed in writing eqs. (2) and (3).

Of the various couplings occurring in eqs. (2) and (3), those of $\tilde{S}_{1}$ and $\tilde{R}_{2}$ do not contribute to $\tau$ and $t$ DFF's in the limit of massless neutrinos and $b$ quark, and so we will not consider these.

The low-energy constraints arising from decays of pseudoscalar mesons are very stringent, unless the leptoquark couplings to the light quarks are chiral. Hence many authors assume couplings to be either left handed or right handed. However, we are going to consider only the third generation leptoquarks on whose couplings there are no strong limits from meson decays. So we need not assume their couplings to be chiral. More importantly, we need both left- and right-handed couplings to be present for the EDFF and WDFF to be nonzero. If, however, the third generation leptoquarks mix substantially with those of the first and second generations, the low-energy constraints would apply more or less unchanged. We will therefore assume mixing to be absent. 


\begin{tabular}{||c|c|c|c|c||}
\hline & $S U(2)_{L}$ & $U(1)$ & $S U(3)_{c}$ & $Q$ \\
\hline$S_{1}$ & 1 & $\frac{1}{3}$ & $3^{*}$ & $\frac{1}{3}$ \\
$\tilde{S}_{1}$ & 1 & $\frac{4}{3}$ & $3^{*}$ & $\frac{4}{3}$ \\
$S_{3}$ & 3 & $\frac{1}{3}$ & $3^{*}$ & $\frac{4}{3}, \frac{1}{3}, \frac{-2}{3}$ \\
$R_{2}$ & 2 & $\frac{7}{6}$ & 3 & $\frac{5}{3}, \frac{2}{3}$ \\
$\tilde{R}_{2}$ & 2 & $\frac{1}{6}$ & 3 & $\frac{2}{3}, \frac{-1}{3}$ \\
\hline
\end{tabular}

Table 1: $S U(2)_{L}, U(1), S U(3)_{c}$ and electric charge assignments of the various leptoquarks

While only one of the components of each leptoquark multiplets would contribute to the form factors we calculate, it is important to note that the constraints we will use on the leptoquark couplings and masses were derived assuming that leptoquarks within a multiplet are degenerate 15, 16, 17]. These constraints were also derived under the assumption of chiral couplings for leptoquarks. However, in the presence of both left-handed and right-handed couplings, the constraints are expected to be somewhat more stringent. We have, however, chosen the constraints on left-handed couplings for our analysis, since these are more stringent.

The couplings of leptoquarks to a single $\gamma$ or $Z$ is given by

$$
\mathcal{L}_{\gamma, Z}=-i e \sum_{i} \phi_{i}^{\dagger} \stackrel{\leftrightarrow}{\partial}_{\mu} \phi_{i}\left[Q_{i} A^{\mu}-\frac{T_{3 L}-Q_{i} s_{W}^{2}}{s_{W} c_{W}} Z^{\mu}\right],
$$

where $\phi_{i}$ are the various scalar fields, $T_{3 i}$ and $Q_{i}$ are the respective values of the third component of weak isospin and electric charge, and $c_{W}=\cos \theta_{W}$, $s_{W}=\sin \theta_{W}, \theta_{W}$ being the weak mixing angle.

We use the couplings written down in eqs. (2), (3) and (4) to obtain expressions for the EDFF and WDFF of $\tau$ and $t$ at the one-loop level in the next section. 

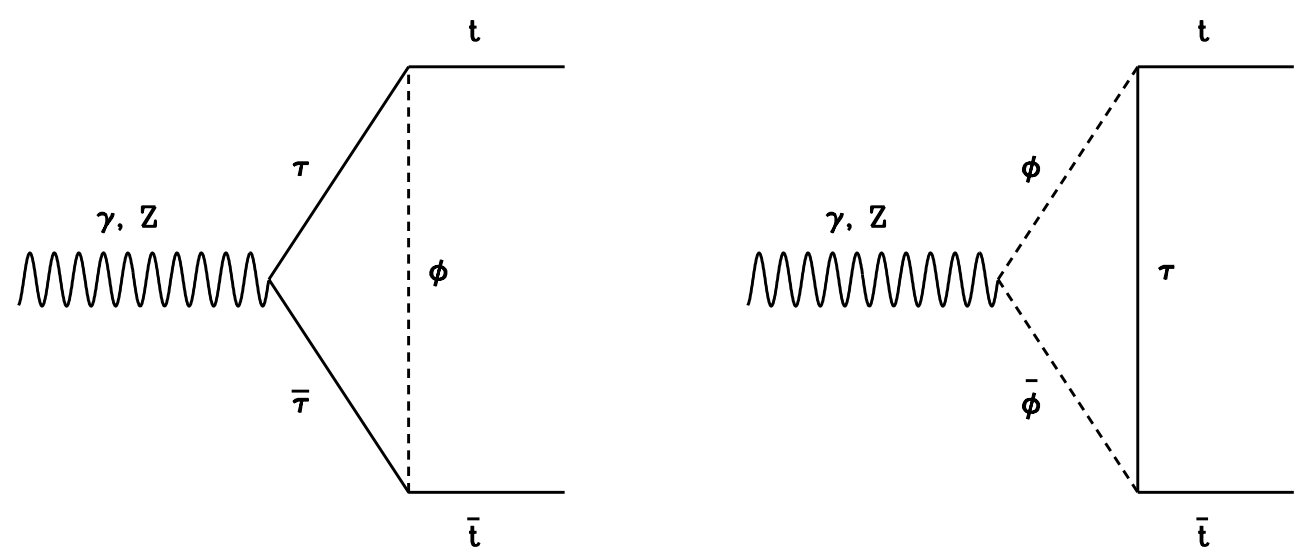

Figure 1: Feynman diagrams corresponding to one-loop correction to the $t \bar{t}(\gamma, Z)$ vertex in the presence of a third generation leptoquark.

\section{Electric and weak dipole form factors}

Using the leptoquark couplings described in the last section we shall look at the one-loop corrections to the $t \bar{t}(\gamma, Z)$ vertices. We use Cutkosky rules [21] to calculate the imaginary part of the DFF and from this the real part is obtained using a dispersion relation.

\subsection{EDFF and WDFF of the top quark}

At one-loop level the diagrams giving rise to the correction to the $t \bar{t}(\gamma, Z)$ coupling due to the leptoquark are given in Fig. 1. We use the symbol $\phi$ for the generic leptoquark.

The lepton could be $\nu_{\tau}$ instead of $\tau$ interacting with a leptoquark of different $T_{3}$ and $Q$ values. However, as we shall see, the contribution to the CP-violating DFF's turns out to be proportional to the mass of the virtual lepton because of chirality flip in scalar couplings. In the limit of small $\nu_{\tau}$ mass, only the $\tau$ contribution is present.

We use Cutkosky rules to calculate the absorptive part of the process. This means considering intermediate particles to be on shell and hence the conditions on the $\gamma / Z$ boson momentum $q$ for diagram in Fig. 1 are $q^{2}>4 m_{\tau}^{2}$ and $q^{2}>4 m_{\phi}^{2}$ in case of $\tau$ pair production and leptoquark pair production respectively, where $m_{\phi}$ is the leptoquark mass. We calculate the vertex contribution coming from these two diagrams.

Using the couplings given in eqs. (2) and (3) we obtain the imaginary 
part of the top quark EDFF and WDFF as

$$
\begin{aligned}
\operatorname{Im} d_{t}^{\gamma}(s)= & \frac{e g_{\phi}^{2}}{4 \pi s} m_{\tau} \operatorname{Im}\left(a^{*} b\right)\left\{-F_{1}^{t}(s)+Q F_{2}^{t}(s)\right\} \\
\operatorname{Im} d_{t}^{Z}(s)= & \frac{e g_{\phi}^{2}}{4 \pi s \sin \theta_{W} \cos \theta_{W}} m_{\tau} \operatorname{Im}\left(a^{*} b\right) \\
& \times\left\{\frac{1}{2}\left(-\frac{1}{2}+2 \sin ^{2} \theta_{W}\right) F_{1}^{t}(s)+\left(T_{3}-Q \sin ^{2} \theta_{W}\right) F_{2}^{t}(s)\right\}
\end{aligned}
$$

where

$$
\begin{aligned}
F_{1}^{t}(s)= & \frac{1}{\beta_{t}^{2}}\left\{\beta_{\tau}+\frac{1}{s \beta_{t}}\left(m_{t}^{2}+m_{\phi}^{2}-m_{\tau}^{2}\right)\right. \\
& \left.\times \ln \left[\frac{2\left(m_{t}^{2}+m_{\tau}^{2}-m_{\phi}^{2}\right)-s\left(1-\beta_{t} \beta_{\tau}\right)}{2\left(m_{t}^{2}+m_{\tau}^{2}-m_{\phi}^{2}\right)-s\left(1+\beta_{t} \beta_{\tau}\right)}\right]\right\} \theta\left(s-4 m_{t}^{2}\right),
\end{aligned}
$$

and

$$
\begin{aligned}
F_{2}^{t}(s)= & \frac{1}{\beta_{t}^{2}}\left\{\beta_{\phi}-\frac{1}{s \beta_{t}}\left(m_{t}^{2}+m_{\phi}^{2}-m_{\tau}^{2}-\frac{s}{2}\right)\right. \\
& \left.\times \ln \left[\frac{2\left(m_{t}^{2}-m_{\tau}^{2}+m_{\phi}^{2}\right)-s\left(1-\beta_{t} \beta_{\phi}\right)}{2\left(m_{t}^{2}-m_{\tau}^{2}+m_{\phi}^{2}\right)-s\left(1+\beta_{t} \beta_{\phi}\right)}\right]\right\} \theta\left(s-4 m_{\phi}^{2}\right) .
\end{aligned}
$$

In the above equation, $T_{3}$ and $Q$ refer respectively to the third component of isospin and charge of the leptoquark $\phi, g_{\phi}$ is the absolute value of the coupling constant, assuming $\left|g_{L}\right|=\left|g_{R}\right|$ (and $\left|h_{2 L}\right|=\left|h_{2 R}\right|$ ) occurring in eqs. (2) and (3), and $a$ and $b$ are phase factors of the corresponding vector and axial vector couplings. $\beta_{t}, \beta_{\tau}$ and $\beta_{\phi}$ refer to the velocities of $t, \tau$ and $\phi$,

$$
\beta_{t, \tau, \phi}=\sqrt{1-\frac{4 m_{t, \tau, \phi}^{2}}{s}} .
$$

The expression in eq. (6) is valid for $s>4 m_{t}^{2}$. $F_{1}^{t}(s)$ for $2 m_{\tau}<\sqrt{s}<2 m_{t}$ is given by the analytic continuation of eq. (6):

$$
\begin{aligned}
F_{1}^{t}(s)= & \frac{1}{\beta_{t}^{2}}\left\{\beta_{\tau}+\frac{2}{s \sqrt{-\beta_{t}^{2}}}\left(m_{t}^{2}+m_{\phi}^{2}-m_{\tau}^{2}\right)\right. \\
& \left.\times \tan ^{-1}\left[\frac{\sqrt{-\beta_{t}^{2}} \beta_{\tau} s}{2\left(m_{t}^{2}+m_{\tau}^{2}-m_{\phi}^{2}\right)-s}\right]\right\} \theta\left(s-4 m_{\tau}^{2}\right) \theta\left(4 m_{t}^{2}-s\right)
\end{aligned}
$$


The real parts of the form factors are obtained using an unsubtracted dispersion relation

$$
\operatorname{Re} d_{t}^{\gamma, Z}(s)=\frac{\mathrm{P}}{\pi} \int_{4 m_{\tau}^{2}}^{\infty} \frac{\operatorname{Im} d_{t}^{\gamma, Z}\left(s^{\prime}\right)}{s^{\prime}-s} d s^{\prime},
$$

where $P$ denotes the principal part of the integral.

It can be seen that the dispersion integrals are convergent and do not need any subtraction. This is to be expected since the dipole form factors, which correspond to dimension 5 operators, should be finite in a renormalizable theory.

\subsection{EDFF and WDFF of the $\tau$ lepton}

The EDFF and WDFF of the tau lepton also arise in the same leptoquark theory from diagrams exactly analogous to the ones in Fig. 1, with the roles of $t$ and $\tau$ interchanged. Proceeding exactly as in the previous section, we obtain expressions for the imaginary parts of the tau lepton EDFF and WDFF, neglecting the $b$ mass.

$$
\begin{aligned}
\operatorname{Im} d_{\tau}^{\gamma}(s)= & \frac{3 e g_{\phi}^{2}}{4 \pi s} m_{t} \operatorname{Im}\left(a^{*} b\right)\left\{\frac{2}{3} F_{1}^{\tau}(s)-Q F_{2}^{\tau}(s)\right\} \\
\operatorname{Im} d_{\tau}^{Z}(s)= & \frac{3 e g_{\phi}^{2}}{4 \pi s \sin \theta_{W} \cos \theta_{W}} m_{t} \operatorname{Im}\left(a^{*} b\right) \\
& \times\left\{\frac{1}{2}\left(\frac{1}{2}-\frac{4}{3} \sin ^{2} \theta_{W}\right) F_{1}^{\tau}(s)-\left(T_{3}-Q \sin ^{2} \theta_{W}\right) F_{2}^{\tau}(s)\right\}
\end{aligned}
$$

where

$$
\begin{aligned}
F_{1}^{\tau}(s)= & \frac{1}{\beta_{\tau}^{2}}\left\{\beta_{t}+\frac{1}{s \beta_{\tau}}\left(m_{\tau}^{2}+m_{\phi}^{2}-m_{t}^{2}\right)\right. \\
& \left.\times \ln \left[\frac{2\left(m_{\tau}^{2}+m_{t}^{2}-m_{\phi}^{2}\right)-s\left(1-\beta_{t} \beta_{\tau}\right)}{2\left(m_{\tau}^{2}+m_{t}^{2}-m_{\phi}^{2}\right)-s\left(1+\beta_{t} \beta_{\tau}\right)}\right]\right\} \theta\left(s-4 m_{t}^{2}\right),
\end{aligned}
$$

and

$$
\begin{aligned}
F_{2}^{\tau}(s)= & \frac{1}{\beta_{\tau}^{2}}\left\{\beta_{\phi}-\frac{1}{s \beta_{\tau}}\left(m_{\tau}^{2}+m_{\phi}^{2}-m_{t}^{2}-\frac{s}{2}\right)\right. \\
& \left.\times \ln \left[\frac{2\left(m_{\tau}^{2}-m_{t}^{2}+m_{\phi}^{2}\right)-s\left(1-\beta_{\tau} \beta_{\phi}\right)}{2\left(m_{\tau}^{2}-m_{t}^{2}+m_{\phi}^{2}\right)-s\left(1+\beta_{\tau} \beta_{\phi}\right)}\right]\right\} \theta\left(s-4 m_{\phi}^{2}\right) .
\end{aligned}
$$



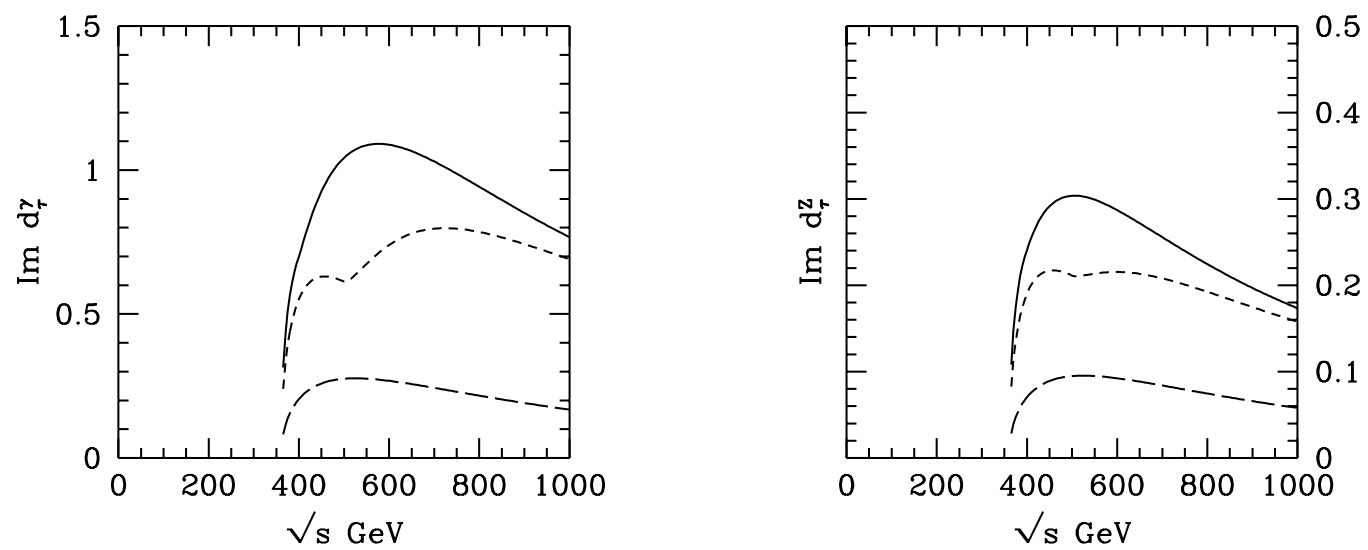

Figure 2: Imaginary parts of the electric (figure on the left) and weak (figure on the right) dipole form factors of $\tau$ in units of $10^{-18} e \mathrm{~cm}$ as functions of c.m. energy $\sqrt{s}$ for the model with leptoquark $R_{2}$. Solid, dashed and dash-dotted lines correspond to leptoquark masses of $200 \mathrm{GeV}, 250 \mathrm{GeV}$ and $500 \mathrm{GeV}$ respectively. $g_{\phi}$ is chosen to be 1 .

Since both $m_{t}$ and $m_{\phi}$ are larger than $m_{\tau}$, there is no domain where an analytic continuation is needed.

As before, the real parts of the form factors are given by the unsubstracted dispersion relations:

$$
\operatorname{Re} d_{\tau}^{\gamma, Z}(s)=\frac{\mathrm{P}}{\pi} \int_{4 m_{\tau}^{2}}^{\infty} \frac{\operatorname{Im} d_{\tau}^{\gamma, Z}\left(s^{\prime}\right)}{s^{\prime}-s} d s^{\prime}
$$

In the next section we will evaluate the real and imaginary parts of the $t$ and $\tau$ form factors numerically for different choices of masses and couplings of the leptoquarks. Using the experimental limits on the tau lepton DFF's we obtain bounds on the masses and couplings of the leptoquarks.

\section{Numerical results}

We use here the expressions of the previous section to get numerical values for the various form factors. While we have analytic expressions for the imaginary parts, the real parts, given by the dispersion integrals, have been evaluated by numerical integration.

To investigate how large the form factors can be, consistent with LEP constraints, we have plotted the $\tau$ and $t$ form factors as functions of $\sqrt{s}$. We 

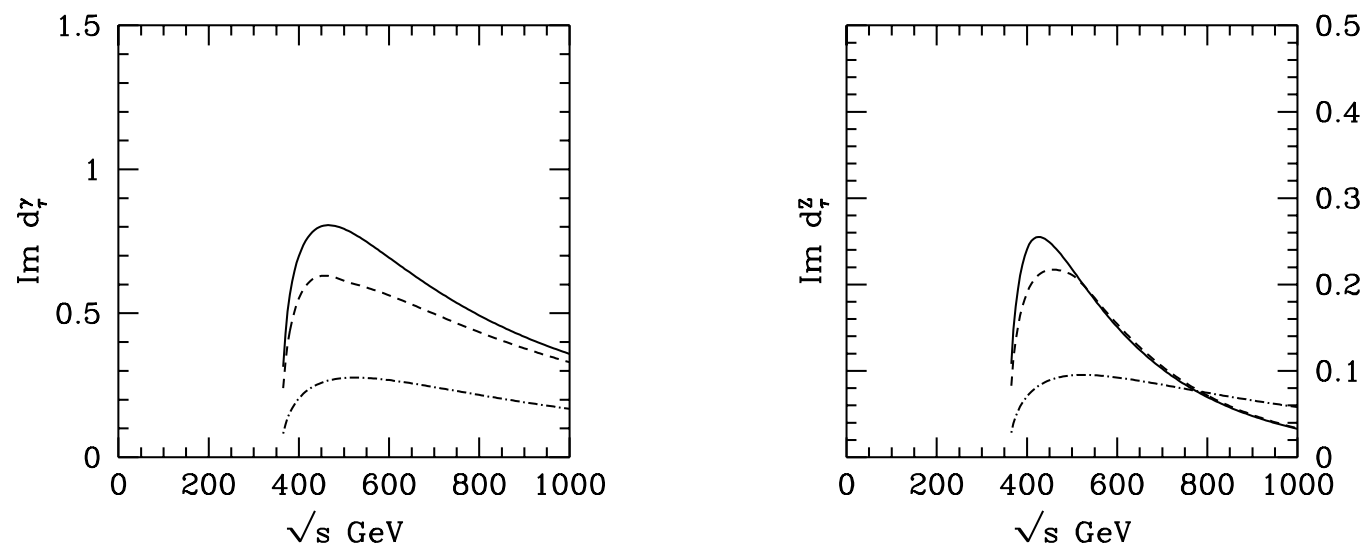

Figure 3: Imaginary parts of the electric (figure on the left) and weak (figure on the right) dipole form factors of $\tau$ in units of $10^{-18} e \mathrm{~cm}$ as functions of c.m. energy $\sqrt{s}$ for the model with leptoquark $S_{1}$. Solid, dashed and dash-dotted lines correspond to leptoquark masses of $200 \mathrm{GeV}, 250 \mathrm{GeV}$ and $500 \mathrm{GeV}$ respectively. $g_{\phi}$ is chosen to be 1 .
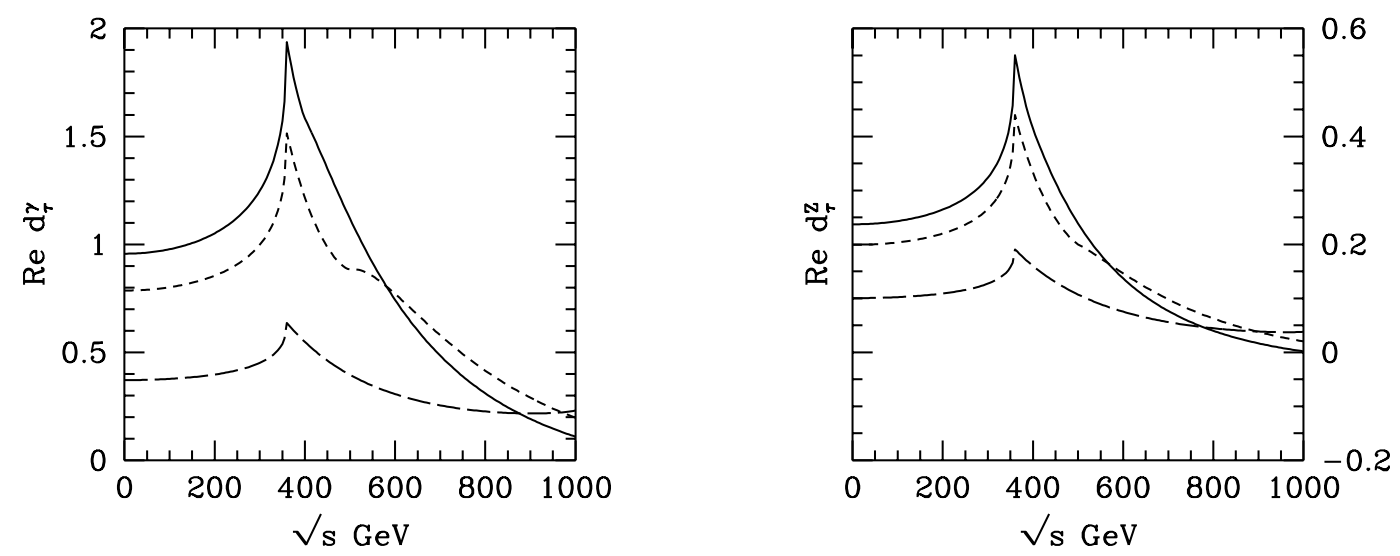

Figure 4: Real parts of the electric (figure on the left) and weak (figure on the right) dipole form factors of $\tau$ in units of $10^{-18} e \mathrm{~cm}$ as functions of c.m. energy $\sqrt{s}$ for the model with leptoquark $R_{2}$. Solid, dashed and dash-dotted lines correspond to leptoquark masses of $200 \mathrm{GeV}, 250 \mathrm{GeV}$ and $500 \mathrm{GeV}$ respectively. $g_{\phi}$ is chosen to be 1 . 

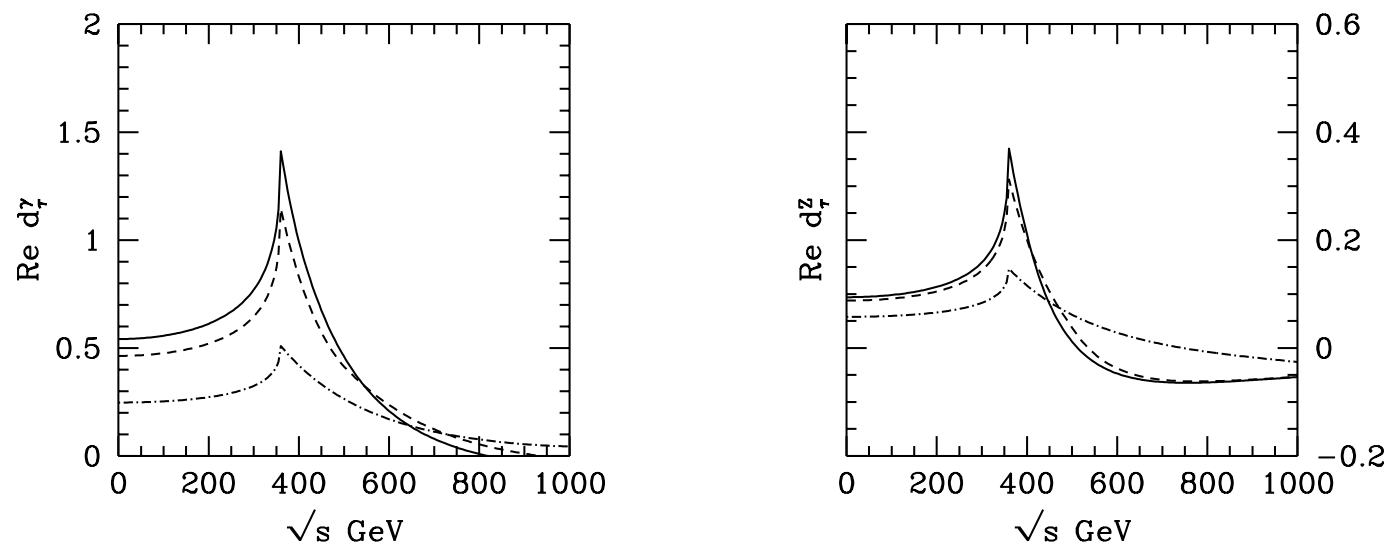

Figure 5: Real parts of the electric (figure on the left) and weak (figure on the right) dipole form factors of $\tau$ in units of $10^{-18} e \mathrm{~cm}$ as functions of c.m. energy $\sqrt{s}$ for the model with leptoquark $S_{1}$. Solid, dashed and dash-dotted lines correspond to leptoquark masses of $200 \mathrm{GeV}, 250 \mathrm{GeV}$ and $500 \mathrm{GeV}$ respectively. $g_{\phi}$ is chosen to be 1 .

have chosen $m_{t}=180 \mathrm{GeV}$ and a maximal value $\operatorname{Im}\left(a^{*} b\right)=1 / 2$. We have considered three different leptoquark masses, viz., $200 \mathrm{GeV}, 250 \mathrm{GeV}$ and $500 \mathrm{GeV} . g_{\phi}$ is chosen to be 1 . In all cases dipole form factors are larger for higher leptoquark masses upto around c.m. energy of $500 \mathrm{GeV}$. At higher energies dependence on the mass becomes weaker than that at lower energies. Among the leptoquarks belonging to the three representations considered, $R_{2}$ (see Table 11 for quantum numbers) gives the largest form factors. The leptoquarks $S_{3}$ (isospin triplet) and $S_{1}$ (isospin singlet) give the same values for DFF's except for the sign, and so we have presented only the results for the $S_{1}$ model.

Figs. 2 and 3 show the dependence of the imaginary parts of the $\tau$ EDFF and WDFF on the c.m. energy. Similarly, Figs. 1 and 1 show the dependence of the real parts. Since the top quark mass is assumed to be $180 \mathrm{GeV}$, there is a peak at $360 \mathrm{GeV}$. A similar, but not so prominent, behaviour is seen at the leptoquark threshold.

Figs. 6 and 7 show the variation of the imaginary part of the $t$ DFF's with c.m. energy, and figs. 8 and 9 show the corresponding curves for the real parts". The behaviour is similar to that in the tau case with peaks

\footnotetext{
${ }^{1}$ Note that in the case of the top quark, the figures corresponding imaginary and real parts of EDFF, and real part of WDFF, are plotted at in two parts, with different scales
} 

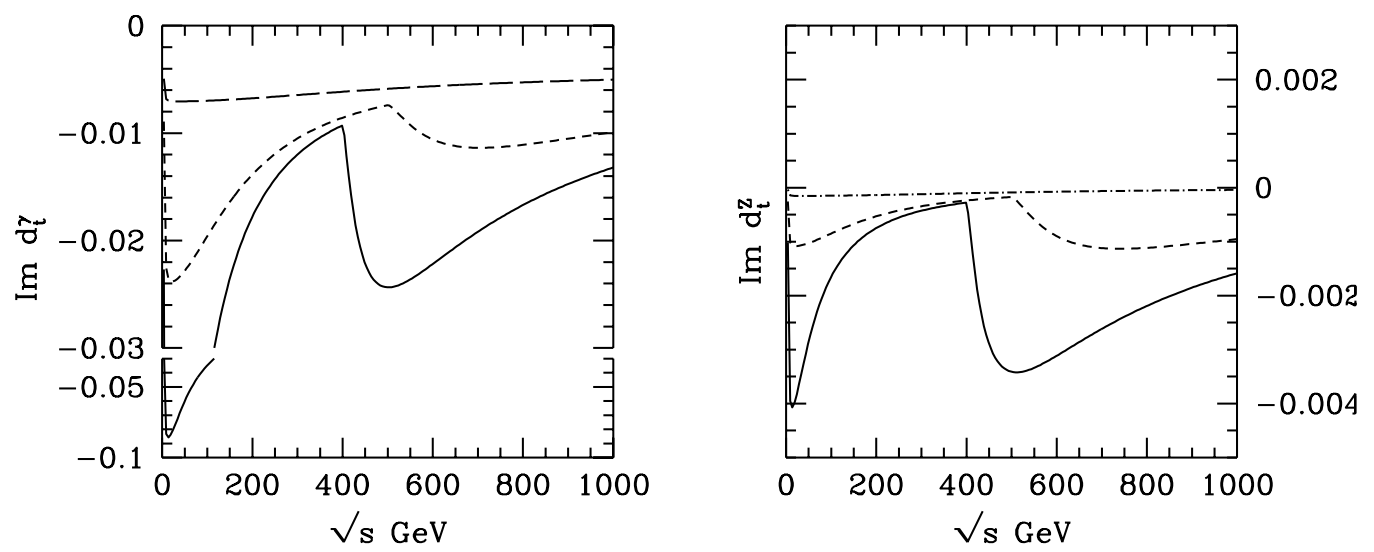

Figure 6: Imaginary parts of the electric (figure on the left) and weak (figure on the right) dipole form factors of the top quark in units of $10^{-18} \mathrm{e} \mathrm{cm}$ as functions of c.m. energy $\sqrt{s}$ for the model with leptoquark $R_{2}$. Solid, dashed and dashdotted lines correspond to leptoquark masses of $200 \mathrm{GeV}, 250 \mathrm{GeV}$ and $500 \mathrm{GeV}$ respectively. $g_{\phi}$ is chosen to be 1 .

at $\tau$ and leptoquark resonances. In case of the top quark form factors, it is interesting to observe the difference in signs, especially of the WDFF, between the $R_{2}$ and $S_{1}$ leptoquark models.

It is seen from the curves that in general, the $R_{2}$ model gives larger values of the form factors compared to the $S_{1}$ model. We therefore concentrate on the $R_{2}$ model in what follows.

At a fixed c.m. energy the form factors are functions of two parameters - the mass and the coupling of the leptoquark considered. From an assumed value of the form factor it is possible to get contours in the plane of the mass and coupling constant of the leptoquark. Contours in the mass-coupling plane for the doublet $R_{2}$ leptoquark model are given for different values of tau lepton DFF's in Figs. 10 and 11. Validity of perturbation theory allows values of coupling $g_{\phi}<4 \pi$. In the case of the tau lepton we have considered the present experimental limits on the dipole moments.

Fig. 11 shows the allowed region in the $m_{\phi^{-}} g_{\phi}$ plane which lies below the solid line if we consider the present experimental limit on the tau electric dipole moment. No value of $m_{\phi}$ or $g_{\phi}$ is completely excluded. However, it is possible that future experiments may give more stringent limits on dipole

for the $y$ axis in the two parts. 

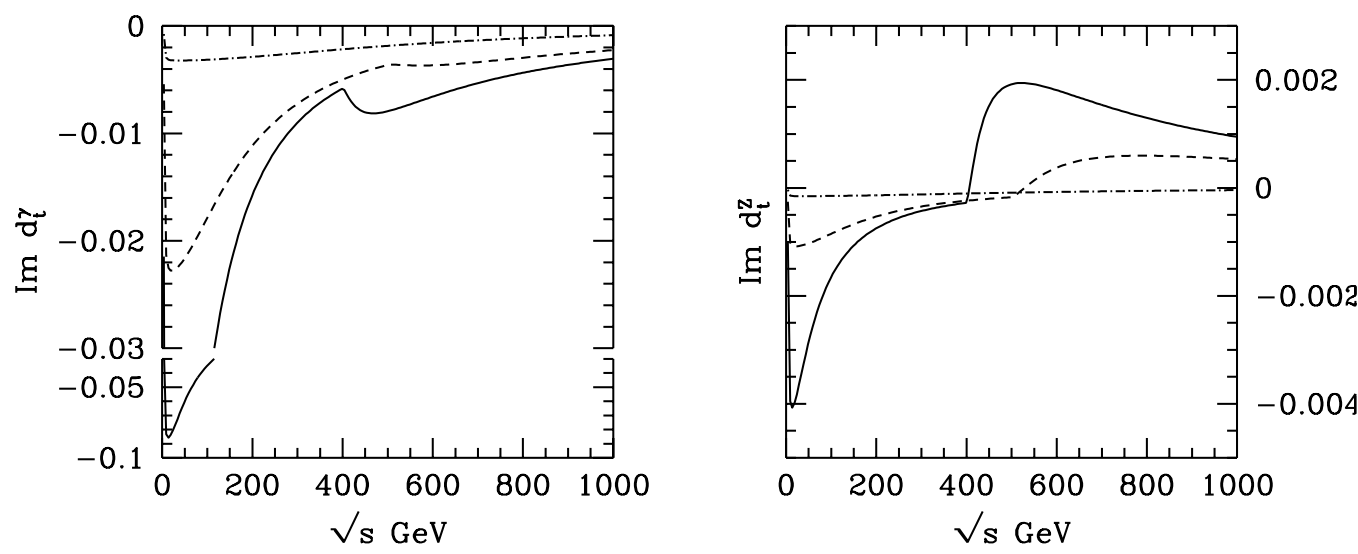

Figure 7: Imaginary parts of the electric (figure on the left) and weak (figure on the right) dipole form factors of the top quark in units of $10^{-18} \mathrm{e} \mathrm{cm}$ as functions of c.m. energy $\sqrt{s}$ for the model with leptoquark $S_{1}$. Solid, dashed and dashdotted lines correspond to leptoquark masses of $200 \mathrm{GeV}, 250 \mathrm{GeV}$ and $500 \mathrm{GeV}$ respectively. $g_{\phi}$ is chosen to be 1 .
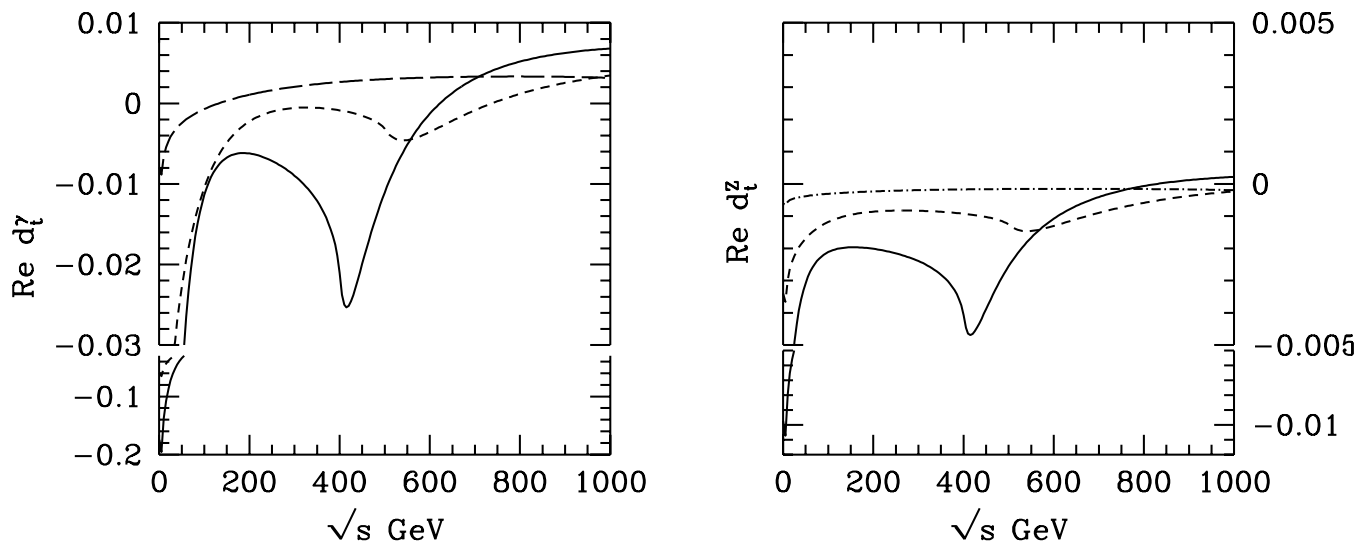

Figure 8: Real parts of the electric (figure on the left) and weak (figure on the right) dipole form factors of the top quark in units of $10^{-18} \mathrm{e} \mathrm{cm}$ as functions of c.m. energy $\sqrt{s}$ for the model with leptoquark $R_{2}$. Solid, dashed and dashdotted lines correspond to leptoquark masses of $200 \mathrm{GeV}, 250 \mathrm{GeV}$ and $500 \mathrm{GeV}$ respectively. $g_{\phi}$ is chosen to be 1 . 

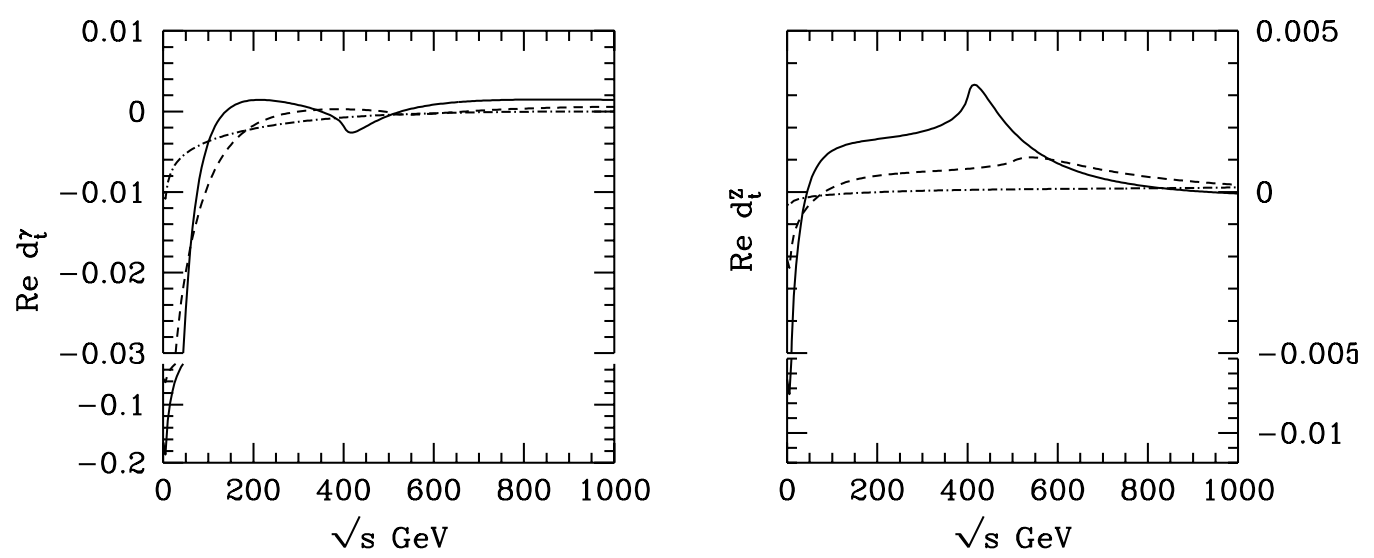

Figure 9: Real parts of the electric (figure on the left) and weak (figure on the right) dipole form factors of the top quark in units of $10^{-18} e \mathrm{~cm}$ as functions of c.m. energy $\sqrt{s}$ for the model with leptoquark $S_{1}$. Solid, dashed and dashdotted lines correspond to leptoquark masses of $200 \mathrm{GeV}, 250 \mathrm{GeV}$ and $500 \mathrm{GeV}$ respectively. $g_{\phi}$ is chosen to be 1 .
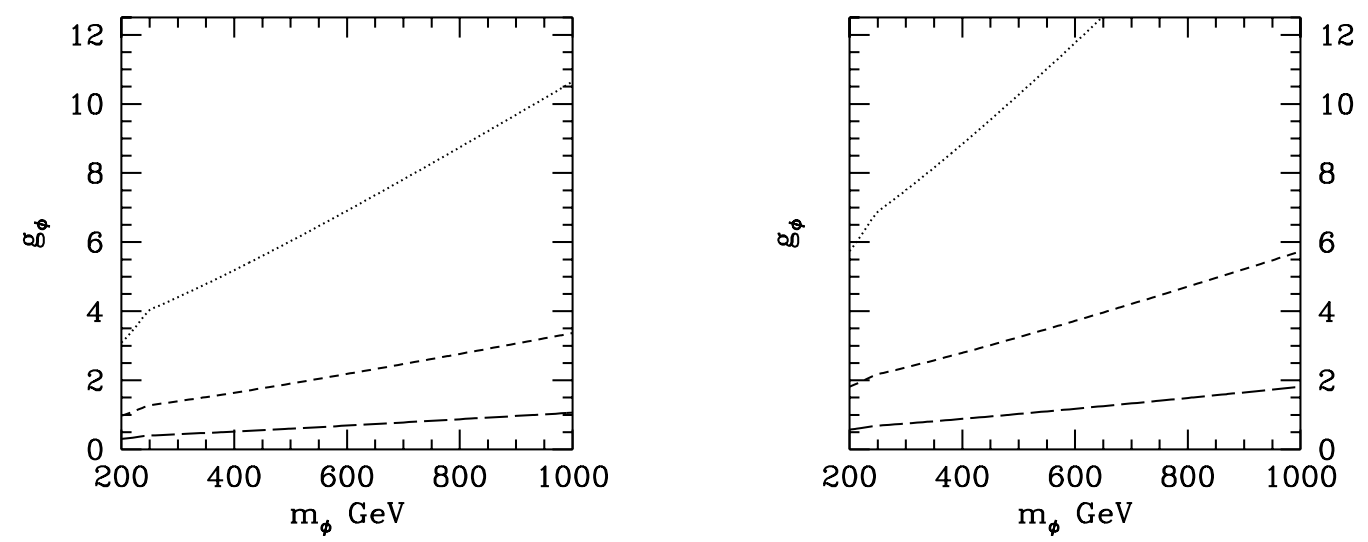

Figure 10: Contours in the mass-coupling plane for different values of imaginary part of EDFF (left) and WDFF (right) of $\tau$ for the $R_{2}$ model. Dotted, dashed, long dashed and dash-dotted lines correspond to DFF values of $10^{-17}, 10^{-18}, 10^{-19}$ and $10^{-20} e \mathrm{~cm}$ respectively. A c.m. energy of $500 \mathrm{GeV}$ is assumed. 

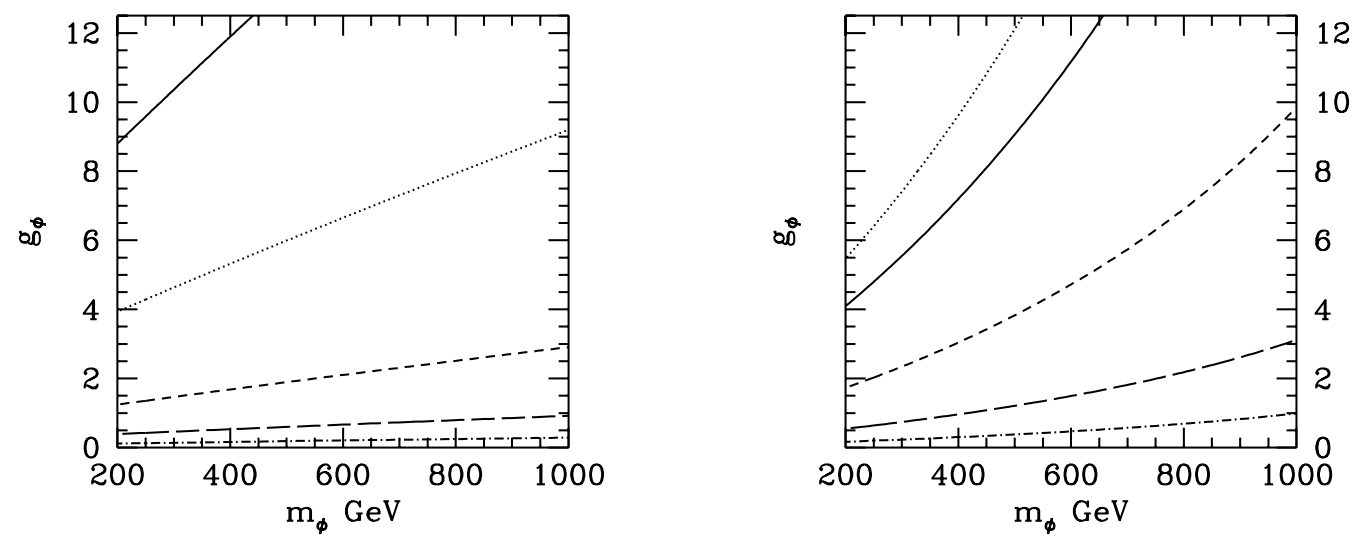

Figure 11: Contours in the mass-coupling plane for different values of the real parts of EDFF (left) and WDFF (right) of $\tau$ for the $R_{2}$ model. Type of lines and corresponding DFF values are the same as those in Fig. 10. The solid curve corresponds to the experimental bounds on the dipole momenta, which are $d_{\tau}^{\gamma}=$ $5 \times 10^{-17} e \mathrm{~cm}, \operatorname{Re} d_{\tau}^{Z}=5.6 \times 10^{-18} e \mathrm{~cm}$. EDFF values are at a c.m. energy of $4 \mathrm{GeV}$ while WDFF values are at $91.18 \mathrm{GeV}$. Top-quark mass is taken to be 180 $\mathrm{GeV}$ in all cases.

form factors which may put upper bounds on the coupling or lower bounds on the mass.

As mentioned before, LEP results on $Z$ partial widths have been used to obtain constraints on masses and couplings for third-generation leptoquarks [15, 16, 17]. We have chosen Éboli's [17 limits to compare with the constraints we get from the dipole form factors. The other limits would give similar results. Fig. 12 shows contours for different values of the real part of the electric dipole form factor of $\tau$ along with the limit obtained by Éboli [17] in the $m_{\phi^{-}} g_{\phi}$ plane. To accommodate their limits we have to restrict the electric dipole form factors of $\tau$ to be smaller than about $10^{-19} \mathrm{ecm}$. A similar analysis shows that the weak dipole form factors must be smaller than about $10^{-20} \mathrm{ecm}$.

The best limits on $m_{\phi}$ and $g_{\phi}$ obtainable from the experimental limits on form factors is that from the real part of the weak dipole moment of the tau lepton, and we use that limit in what follows.

In the case of top quark there are no experimental limits. From the constraints obtained on the mass and the coupling from the experimental 


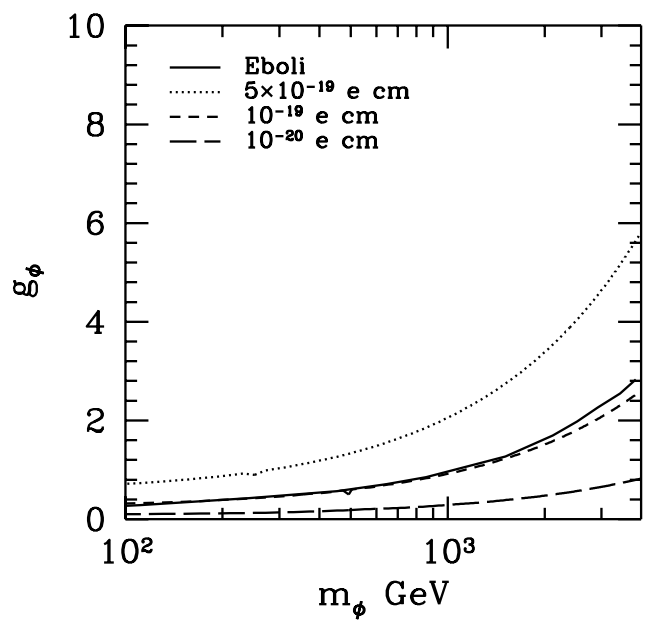

Figure 12: Contours in the mass-coupling plane for the $R_{2}$ leptoquark model. The solid line represents the limits from the one-loop calculation by Éboli [17] while the other curves correspond to different values of the real part of the tau EDFF. Top-quark mass of $175 \mathrm{GeV}$ is assumed. The c.m. energy used is $500 \mathrm{GeV}$.

bound on the real part of the tau WDFF, Figs. 13 and 14 show that the top quark can have values for the imaginary part of the EDFF as high as $10^{-19} e \mathrm{~cm}$ except for a small mass range $(\sim 250-300 \mathrm{GeV})$. The imaginary part of the weak dipole form factor of the top quark is allowed to be almost as high as $10^{-20} e \mathrm{~cm}$. We get more or less the same limits for the real part of both the electric and the weak dipole form factors.

Again, to accommodate constraints on the masses and couplings from Éboli's result we have to have electric dipole form factors of the order of $10^{-22} e \mathrm{~cm}$ or less and weak dipole form factors of the order of $10^{-23} e \mathrm{~cm}$ or less.

\section{Conclusions}

We have analyzed top and tau electric and weak dipole form factors in models with scalar leptoquarks of weak isospin $0, \frac{1}{2}$ and 1 . We calculate form factors for different values of $\sqrt{s}$. In general, for leptoquark couplings in the perturbative region and masses allowed by direct experimental searches, large EDFF and WDFF values for both top and tau are possible. In case of the top, the values can be as high as $10^{-20} e \mathrm{~cm}$, whereas tau form factors 

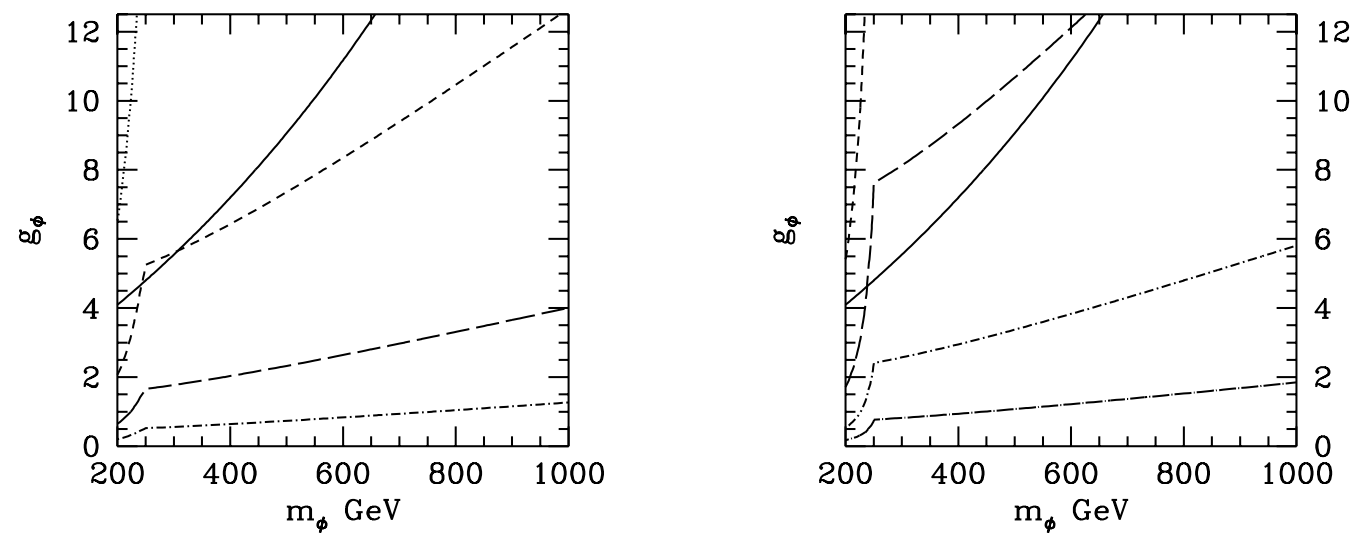

Figure 13: Contours in the mass-coupling plane for different values of imaginary parts of the top EDFF (the figure on the left) and WDFF (the figure on the right) Dotted, dashed, long dashed, dash-dotted and long dash-dotted lines correspond to DFF values of $10^{-18}, 10^{-19}, 10^{-20}, 10^{-21}$ and $10^{-22} \mathrm{e} \mathrm{cm}$ respectively. The solid curve corresponds to the experimental bound on the real part of the weak dipole moment of $\tau, 5.6 \times 10^{-18} e \mathrm{~cm}$. A c.m. energy of $500 \mathrm{GeV}$ and a top-quark mass of $180 \mathrm{GeV}$ are assumed.
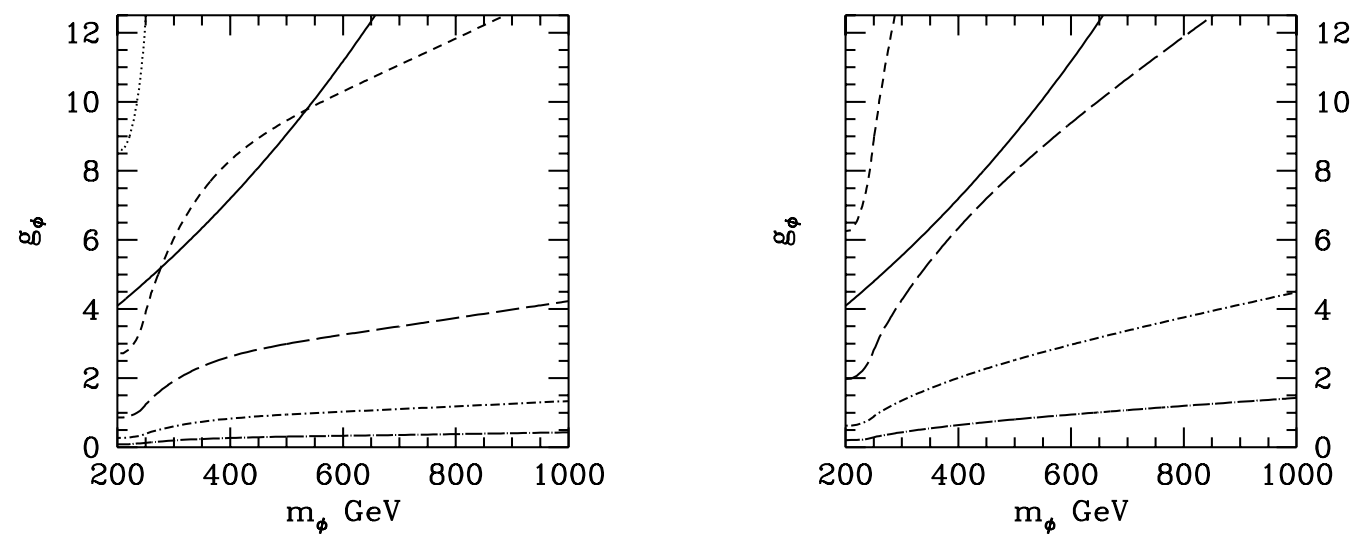

Figure 14: Similar to Fig. 13 but with contours from the real part of DFF's. The solid lines are the same as in the Fig. 13 . 
can be of the order of $10^{-18} e \mathrm{~cm}$. The values of form factors are larger for the case of weak isosopin $\frac{1}{2}$, and we have therefore concentrated on that case.

We also obtain contours in the mass-coupling plane corresponding to fixed values of form factors for a given $\sqrt{s}$, and use the experimental limits from LEP on the tau form factors to obtain the allowed region in the plane. This gives constraints on the top form factors. For most of the range of couplings and leptoquark mass, EDFF of the top can be as large as $10^{-19} e \mathrm{~cm}$, and the WDFF can be as large as $10^{-20} e \mathrm{~cm}$. We have also used the indirect constraints on the mass and coupling of scalar leptoquarks derived from LEP measurements of the one-loop contribution of leptoquarks to the $Z$ partial decay widths. These constraints are more stringent, and do not permit tau EDFF above about $10^{-19} e \mathrm{~cm}$ and tau WDFF above about $10^{-20} e \mathrm{~cm}$. The corresponding upper bounds on the top EDFF and WDFF are, respectively, $10^{-22} e \mathrm{~cm}$ and $10^{-23}$ e $\mathrm{cm}$.

We thus conclude that though large dipole form factors are allowed in the scalar leptoquark model with parameter values consistent with direct experimental constraints, the indirect constraints from one-loop contributions to $Z$ decay parameters seem to permit only values of form factors which lie below the range likely to be explored in experiments in the foreseeable future.

It would be interesting to investigate the one-loop contribution of the third-generation leptoquarks to $\mathrm{CP}$ violation in the decay $t \rightarrow b W$.

Finally, we end with a few comments on comparison of our work with other recent work on DFF in third-generation leptoquark models [6, 7]. Mahanta [6] has estimated the $\tau$ EDFF and has reached the conclusion that it can be as high as $10^{-19} \mathrm{ecm}$ for a choice of $g_{\phi}$ and $m_{\phi}$ consistent with experimental constraints. He does not discuss the momentum dependence

of DFF's. Bernreuther et al. [7] have obtained the $\sqrt{s}$ dependence of the DFF's of $\tau$. Our results are in agreement with theirs when an overall scale error in their curves is taken into account [22]. Neither of [6] and [7] discuss DFF's of both $t$ and $\tau$ in a concerted manner as we have done here.

\section{Acknowledgements}

We acknowledge with thanks the initial collaboration of Torsten Arens. We also thank Frank Cuypers for lending his contour-plotting program. One of us (S.D.R.) thanks Prof. J.W.F. Valle for his warm hospitality at the 
University of Valencia where he spent a sabbatical year. This work was supported by DGICYT under grant PB95-1077 and by the TMR network grant ERBFMRXCT960090 of the European Union. S. D. R. was supported by DGICYT sabbatical grant SAB95-0175. 


\section{References}

[1] ALEPH collab., D. Busculic et al., Phys. Lett. B 297 (1992) 459; Phys. Lett. B 346 (1995) 371; OPAL collab., P.D. Acton et al., Phys. Lett. B 281 (1992) 405; OPAL collab., R. Ackers et al., Z. Phys. C 66 (1995) 31.

[2] OPAL collab., K. Ackerstaff et al., Zeit. Phys. C 74 (1997) 403.

[3] B. Ananthanarayan and S.D. Rindani, Phys. Rev. D 51 (1995) 5996.

[4] G.L. Kane, G.A. Ladinsky and C.P. Yuan, Phys. Rev. D 45 (1992) 124; W. Bernreuther, O. Nachtmann, P. Overmann and T. Schröder, Nucl. Phys. B 388 (1992) 53; ibid. B 406 (1993)516 (E); W. Bernreuther, T. Schröder and T.N. Pham, Phys. Lett. B 279 (1992) 389; D. Atwood and A. Soni, Phys. Rev. D 45 (1992) 2405; D. Chang, W.-Y. Keung, and I. Phillips, Nucl. Phys. B 408, (1993) 286; ibid., 429 (1994) 255 (E); W. Bernreuther and P. Overmann, Z. Phys. C 61 (1994) 599, ibid., C 72 (1996) 461; B. Grzadkowski, Phys. Lett. B 305 (1993) 384; A. Pilaftsis and M. Nowakowski, Int. J. Mod. Phys. A 9 (1994) 1997; F. Cuypers and S.D. Rindani, Phys. Lett. B 343 (1995) 333; P. Poulose and S.D. Rindani, Phys. Lett. B 349 (1995) 379; Phys. Lett. B 383 (1996) 212; Phys. Rev. D 54 (1996) 4326; A. Bartl, E. Christova and W. Majerotto, Nucl. Phys. B 460 (1996) 235; ibid., B 465 (1996) 365 (E); D. Atwood and A. Soni, BNL-THY-AS-91996, hep-ph/9609418; JLAB-TH-96-14, hepph/9607481; B. Grzadkowski and Z. Hioki, Nucl. Phys. B 484 (1997) 17; Phys. Lett. B 391 ( 1997) 172.

[5] C.R. Schmidt and M.E. Peskin, Phys. Rev. Lett. 69 (1992) 410; C.R. Schmidt, Phys. Lett. B 293 (1992) 111; J.P. Ma and A. Brandenburg, Z. Phys. C 56 (1992) 97; A. Brandenburg and J.P. Ma, Phys. Lett. B 298 (1993) 211; W. Bernreuther and A. Brandenburg, Phys. Rev. D 49 (1994) 4481; P. Haberl, O. Nachtmann and A. Wilch, Phys. Rev. D 53 (1996) 4875; D. Atwood et al., Phys. Rev. D 54 (1996) 5412;

B. Grzadkowski and B. Lampe, Warsaw preprint UW-IFT-05-97, hepph/9706489.

[6] U. Mahanta, Phys. Rev. D 54 (1996) 3377. 
[7] W. Bernreuther, A. Brandenburg and P. Overmann, Phys. Lett. 391 (1997) 413.

[8] B. Adeva et al., Phys. Lett. B 261 (1992) 169.

[9] J. Hobbs, Talk at XXXII Rencontre de Moriond, March 1977.

[10] F. Abe et al., Phys. Rev. Lett. 78 (1997) 2906.

[11] H1 collab., C. Adloff et al., Z. Phys. C 74 (1997) 191; ZEUS collab., J. Breitweg et al., Z. Phys. C 74 (1997) 207.

[12] G. Altarelli et al., CERN-TH/97-40, hep-ph/9703276; M.A. Doncheski and S. Godfrey, OCIP-C-97-02, hep-ph/9703285; J. Blümlein, Z. Phys. C 74 (1997) 605; DESY-97-105, hep-ph/9706362; J. Kalinowski, R. Ruckl, H. Spiesberger and P.M. Zerwas, Z. Phys. C74 (1997) 595; M. Suzuki, LBL-40111, hep-ph/9703316.

[13] J. Blümlein et al., Mod. Phys. Lett. A 9 (1994) 3007.

[14] M. Hirsch, H.V. Klapdor-Kleingrothaus and S.G. Kovalenko, Phys. Lett. B 378 (1996) 17; U. Mahanta, Phys. Lett. B 337 (1994) 128; A.A. Gvozdev et al., hep-ph/9505229.

[15] G. Bhattacharyya, J. Ellis and K. Sridhar, Phys. Lett. B 336 (1994) 100; 338 (1994) 522 (E).

[16] J.K. Mizukoshi, O.J.P. Éboli and M.C. Gonzalez-Garcia, Nucl. Phys. B 443 (1995) 20.

[17] O.J.P. Éboli, IFUSP 1170, hep-ph/9508342.

[18] L.J. Hall and L.J. Randall, Nucl. Phys. B 274 (1986) 157.

[19] C.Q. Geng, Zeit. Phys. C 48 (1990) 279.

[20] W. Buchmüller and D. Wyler, Phys. Lett. B 177 (1986) 377.

[21] R.E. Cutkosky, J. Math. Phys., 1 (1960) 429.

[22] W. Bernreuther, private communcation. 